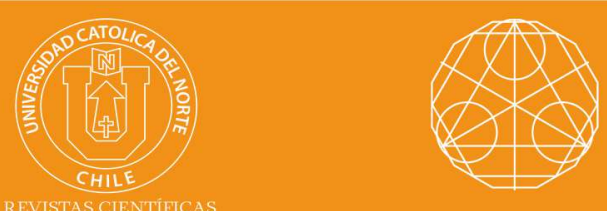

\title{
Neutral stochastic functional differential evolu- tion equations driven by Rosenblatt process with varying-time delays
}

\section{Lakhel El Hassan* iD orcid.org/0000-0002-6615-9706}

*Cadi Ayyad University, National School of Applied Sciences, Safi, Morocco

Q.lakhel@uca.ma

\section{Received: April 2018 | Accepted: July 2019}

\section{Abstract:}

Hermite processes are self-similar processes with stationary increments, the Hermite process of order 1 is fractional Brownian motion and the Hermite process of order 2 is the Rosenblatt process. In this paper we consider a class of time-dependent neutral stochastic functional differential equations with finite delay driven by Rosenblatt process with index $H \in\left(\frac{1}{2}, 1\right)$ which is a special case of a self-similar process with long-range dependence. More precisely, we prove the existence and uniqueness of mild solutions by using stochastic analysis and a fixed-point strategy. Finally, an illustrative example is provided to demonstrate the effectiveness of the theoretical result.

Keywords: Neutral stochastic evolution equations; Evolution operator; Rosenblatt process; Wiener integral; Banach fixed point theorem.

MSC (2010): 60H15, 60G15, 60H20.

\section{Cite this article as (IEEE citation style):}

L. El Hassan,"Neutral stochastic functional differential evolu-

tion equations driven by Rosenblatt process with varying-

time delays", Proyecciones (Antofagasta, On line), vol. 38, no.

4 pp. 653-663, Oct. 2019, doi: 10.22199/issn.0717-6279.

2019-04-0043. [Accessed dd-mm-yyyy].

Article copyright: (c) 2019 Lakhel El Hassan. This is an open access article distributed under the terms of the Creative Commons Licence, which permits unrestricted use and distribution provided the original author and source are credited. 


\section{Introduction}

For the practical applications in the areas such as biology, medicine, physics, finance, electrical engineering, telecommunication networks, and so on, the theory of stochastic evolution equations has attracted research's great interest. For more details, one can see Da Prato and Zabczyk [5], and Ren and Sun [17] and the references therein. In many areas of science, there has been an increasing interest in the investigation of the systems incorporating memory or aftereffect, i.e., there is the effect of delay on state equations. Therefore, there is a real need to discuss stochastic evolution systems with delay. In many mathematical models the claims often display long-range memories, possibly due to extreme weather, natural disasters, in some cases, many stochastic dynamical systems depend not only on present and past states, but also contain the derivatives with delays. Neutral functional differential equations are often used to describe such systems.

Recently, stochastic functional differential equations driven by fractional Brownian motion have attracted the interest of many researchers. One can see $[3,4,6,7,8,9,10]$ and the references therein. Very recently, Lakhel and Mckibeen have discussed the existence of mild solutions for a class of stochastic fractional stochastic differenntial equations driven by a fractional Brownian motion, in Hilbert space, by using the Wiener integral [11]. However, the problem of studing the existence of solution of neutral stochastic differential equations driven by Rosenblatt process is more complicated and still remains open for a while. Hence, techniques and methods for analysing the neutral stochastic fractional stochastic differenntial equations driven by Rosenblatt process should be developed and explored.

On the other hand, the very large utilization of the fractional Brownian motion in practice are due to its self-similarity, stationarity of increments and long-range dependence; one prefers in general $\mathrm{fBm}$ before other processes because it is Gaussian and the calculus for it is easier; but in concrete situations when the gaussianity is not plausible for the model, one can use for example the Rosenblatt process. Although defined during the $60 \mathrm{~s}$ and 70 s $[19,21]$ due to their appearance in the Non-Central Limit Theorem, the systematic analysis of Rosenblatt processes has only been developed during the last ten years, motivated by their nice properties (self-similarity, stationarity of the increments, long-range dependence). Since they are non-Gaussian and self-similar with stationary increments, the Rosenblatt processes can also be an input in models where self-similarity is observed in empirical data which appears to be non-Gaussian. There exists a consis- 
tent literature that focuses on different theoreticalaspects of the Rosenblatt processes. Let us recall some of these works. For example, the rate of convergence to the Rosenblatt process in the Non Central Limit Theorem has been given by Leonenko and Ahn [12]. The analysis of the Rosenblatt process has been investigated by Tudor [22]. The distribution of the Rosenblatt process has been given in [13]. An existence and uniqueness result of mild solutions for a class of neutral stochastic differential equation with infinite delay driven by Rosenblatt process in Hilbert space has been recently established in Sakthivel et al. [18].

Motivated by the above works, this paper is concerned with the existence and uniqueness of mild solutions for a class of time-dependent neutral functional stochastic differential equations driven by non-Gaussian noises, described in the form:

$\left\{\begin{array}{l}d[x(t)+g(t, x(t-r(t)))]=[A(t) x(t)+f(t, x(t-\rho(t)))] d t+\sigma(t) d Z_{H}(t), 0 \leq t \leq T, \\ x(t)=\varphi(t),-\tau \leq t \leq 0,\end{array}\right.$ $(1.1)$

in a real Hilbert space $X$ with inner product $\langle.,$.$\rangle and norm \|$.$\| , where$ $\{A(t), t \in[0, T]\}$ is a family of linear closed operators from a space $X$ into $X$ that generates an evolution system of operators $\{U(t, s), 0 \leq s \leq t \leq$ $T\} . Z_{H}$ is a Rosenblatt process on a real and separable Hilbert space $Y$, $r, \rho:[0,+\infty) \rightarrow[0, \tau](\tau>0)$ are continuous and $f, g:[0,+\infty) \times X \rightarrow$ $X, \quad \sigma:[0,+\infty) \rightarrow \mathcal{L}_{2}^{0}(Y, X), \quad$ are appropriate functions. Here $\mathcal{L}_{2}^{0}(Y, X)$ denotes the space of all $Q$-Hilbert-Schmidt operators from $Y$ into $X$ (see section 2 below).

On the other hand, to the best of our knowledge, there is no paper which investigates the study of time-dependent neutral stochastic functional differential equations with delays driven by Rosenblatt process. Thus, we will make the first attempt to study such problem in this paper.

We organize our paper as follows. Section 2, recapitulate some notations, basic concepts, and basic results about Rosenblatt process, Wiener integral with respect to it over Hilbert spaces and we recall some preliminary results about evolution operator. We need to prove a new technical lemma for the $\mathbf{L}^{2}$-estimate of stochastic convolution integral. Section 3, gives sufficient conditions to prove the existence and uniqueness for the problem (1.1). In Section 4 we give an example to illustrate the efficiency of the obtained result. 


\section{Preliminaries}

In this section we recall some basic results about evolution family, and we introduce the Rosenblatt process as well as the Wiener integral with respect to it. We also establish some important results which will be needed throughout the paper.

\subsection{Evolution families}

In this subsection we introduce the notion of evolution family.

A set $\{U(t, s): 0 \leq s \leq t \leq T\}$ of bounded linear operators on a Hilbert space $X$ is called an evolution family if

(a) $U(t, s) U(s, r)=U(t, r), U(s, s)=I$ if $r \leq s \leq t$,

(b) $(t, s) \rightarrow U(t, s) x$ is strongly continuous for $t>s$.

Let $\{A(t), t \in[0, T]\}$ be a family of closed densely defined linear unbounded operators on the Hilbert space $X$ and with domain $D(A(t))$ independent of $t$, satisfying the following conditions introduced by [1].

There exist constants $\lambda_{0} \geq 0, \theta \in\left(\frac{\pi}{2}, \pi\right), L, K \geq 0$, and $\mu, \nu \in(0,1]$ with $\mu+\nu>1$ such that

$$
\Sigma_{\theta} \cup\{0\} \subset \rho\left(A(t)-\lambda_{0}\right), \quad\left\|R\left(\lambda, A(t)-\lambda_{0}\right)\right\| \leq \frac{K}{1+|\lambda|}
$$

and

(\$1. $\left(2 A(t)-\lambda_{0}\right) R\left(\lambda, A(t)-\lambda_{0}\right)\left[R\left(\lambda_{0}, A(t)\right)-R\left(\lambda_{0}, A(s)\right)\right] \| \leq L|t-s|^{\mu}|\lambda|^{-\nu}$,

for $t, s \in \mathbf{R}, \lambda \in \Sigma_{\theta}$ where $\Sigma_{\theta}:=\{\lambda \in \mathbf{C}-\{0\}:|\arg \lambda| \leq \theta\}$.

It is well known, that this assumption implies that there exists a unique evolution family $\{U(t, s): 0 \leq s \leq t \leq T\}$ on $X$ such that $(t, s) \rightarrow U(t, s) \in$ $L(X)$ is continuous for $t>s, U(\cdot, s) \in \mathcal{C}^{1}((s, \infty), L(X)), \partial_{t} U(t, s)=$ $A(t) U(t, s)$, and

$$
\left\|A(t)^{k} U(t, s)\right\| \leq C(t-s)^{-k}
$$

for $0<t-s \leq 1, k=0,1,0 \leq \alpha<\mu, x \in D\left(\left(\lambda_{0}-A(s)\right)^{\alpha}\right)$, and a constant $C$ depending only on the constants in (2.1)-(2.2). Moreover, $\partial_{s}^{+} U(t, s) x=$ $-U(t, s) A(s) x$ for $t>s$ and $x \in D(A(s))$ with $A(s) x \in \overline{D(A(s))}$. We 
say that $A(\cdot)$ generates $\{U(t, s): 0 \leq s \leq t \leq T\}$. Note that $U(t, s)$ is exponentially bounded by (2.3) with $k=0$.

If $\{A(t), t \in[0, T]\}$ is a second order differential operator $A$, that is $A(t)=A$ for each $t \in[0, T]$, then $A$ generates a $C_{0}$-semigroup $\left\{e^{A t}, t \in\right.$ $[0, T]\}$.

For additional details on evolution system and their properties, we refer the reader to [15].

\subsection{Rosenblatt process}

In this section, we collect some definitions and lemmas on Wiener integrals with respect to an infinite dimensional Rosenblatt process and we recall some basic results about analytical semi-groups and fractional powers of their infinitesimal generators, which will be used throughout the whole of this paper.

For details of this section, we refer the reader to $[22,15]$ and references therein.

Let $(\Omega, \mathcal{F}, \mathbf{P})$ be a complete probability space. Selfsimilar processes are invariant in distribution under suitable scaling. They are of considerable interest in practice since aspects of the selfsimilarity appear in different phenomena like telecommunications, turbulence, hydrology or economics. A self-similar processes can be defined as limits that appear in the so-called Non-Central Limit Theorem (see [21]). We briefly recall the Rosenblatt process as well as the Wiener integral with respect to it.

Consider $\left(\zeta_{n}\right)_{n \in \mathbf{Z}}$ a stationary Gaussian sequence with mean zero and variance 1 which exhibits long range dependence in the sense that the correlation function satisfies

$$
r(n)=\left(\zeta_{0} \zeta_{n}\right)=n^{\frac{2 H-2}{k}} L(n)
$$

with $H \in\left(\frac{1}{2}, 1\right)$ and $\mathrm{L}$ is a slowly varying function at infinity. Let us recall the notion of Hermite rank. Denote by $H_{j}(x)$ the Hermite polynomial of degree $j$ given by $H_{j}=(-1)^{j} e^{\frac{x^{2}}{2}} \frac{d^{j}}{d x^{j}} e^{\frac{-x^{2}}{2}}$ and let $g$ be a function on $\mathbf{R}$ such that $\left[g\left(\zeta_{0}\right)\right]=0$ and $\left[g\left(\zeta_{0}\right)^{2}\right]<\infty$. Assume that $g$ has the following expansion in Hermite polynomials 


$$
g(x)=\sum_{j \geq 0} c_{j} H_{j}(x)
$$

where $c_{j}=\frac{1}{j !}\left(g\left(\zeta_{0} H_{j}\left(\zeta_{0}\right)\right)\right)$. The Hermite rank of $\mathrm{g}$ is defined by

$$
k=\min \left\{j \mid c_{j} \neq 0\right\} .
$$

Since $\left[g\left(\zeta_{0}\right)\right]=0$, we have $k \geq 1$. The following family of stochastic processes

$$
\frac{1}{n^{H}} \sum_{j=1}^{[n t]} g\left(\zeta_{j}\right)
$$

converges as $n \longrightarrow \infty$, in the sense of finite dimensional distributions, to the selfsimilar stochastic process with stationary increments

$\left(2.4 Z_{H}^{k}(t)=c(H, k) \int_{\mathbf{R}^{k}}\left(\int_{0}^{t} \prod_{j=1}^{k}\left(s-y_{j}\right)_{+}^{-\left(\frac{1}{2}+\frac{1-H}{k}\right)} d s\right) d B\left(y_{1}\right) \ldots d B\left(y_{k}\right)\right.$,

where $x_{+}=\max (x, 0)$. The above integral is a Wiener-Itô multiple integral of order $k$ with respect to the standard Brownian motion $(B(y))_{y \in \mathbf{R}}$ and the constant $c(H, k)$ is a normalizing constant that ensures $\left(Z_{H}^{k}(1)\right)^{2}=1$.

The process $\left(Z_{H}^{k}(t)\right)_{t \geq 0}$ is called the Hermite process. When $k=1$ the process given by (2.4) is nothing else that the fractional Brownian motion (fBm) with Hurst parameter $H \in\left(\frac{1}{2}, 1\right)$. For $k=2$ the process is not Gaussian. If $k=2$ then the process (2.4) is known as the Rosenblatt process. It was introduced by Rosenblatt in [19] and was given its name by Taqqu in [20]. The fractional Brownian motion is of course the most studied process in the class of Hermite processes due to its significant importance in modelling. A stochastic calculus with respect to it has been intensively developed in the last decade. The Rosenblatt process is, after fBm, the most well known Hermite process.

We also recall the following properties of the Rorenblatt process:

- The process $Z_{H}^{k}$ is H-selfsimilar in the sense that for any $c>0$,

$$
\left(Z_{H}^{k}(c t)\right)={ }^{(d)}\left(c^{H} Z_{H}^{k}(t)\right),
$$


where $"={ }^{(d)}$ " means equivalence of all finite dimensional distributions. It has stationary increments and all moments are finite.

- From the stationarity of increments and the self-similarity, it follows that, for any $p \geq 1$

$$
\left|Z_{H}(t)-Z_{H}(s)\right|^{p} \leq\left|\left(Z_{H}(1)\right)\right|^{p}|t-s|^{p H} .
$$

As a consequence the Rosenblatt process has Hölder continuous paths of order $\gamma$ with $0<\gamma<H$.

Self-similarity and long-range dependence make this process a useful driving noise in models arising in physics, telecommunication networks, finance and other fields. Consider a time interval $[0, T]$ with arbitrary fixed horizon $T$ and let $\left\{Z_{H}(t), t \in[0, T]\right\}$ the one-dimensional Rosenblatt process with parameter $H \in(1 / 2,1)$. By Tudor [22], it is well known that $Z_{H}$ has the following integral representation:

$$
\left(\mathbb{Z}\left(6_{1}\right)(t)=d(H) \int_{0}^{t} \int_{0}^{t}\left[\int_{y_{1} \vee y_{2}}^{t} \frac{\partial K^{H^{\prime}}}{\partial u}\left(u, y_{1}\right) \frac{\partial K^{H^{\prime}}}{\partial u}\left(u, y_{2}\right) d u\right] d B\left(y_{1}\right) d B\left(y_{2}\right)\right.
$$

where $B=\{B(t): t \in[0, T]\}$ is a Wiener process, $H^{\prime}=\frac{H+1}{2}$ and $K^{H}(t, s)$ is the kernel given by

$$
K^{H}(t, s)=c_{H} s^{\frac{1}{2}-H} \int_{s}^{t}(u-s)^{H-\frac{3}{2}} u^{H-\frac{1}{2}} d u
$$

for $t>s$, where $c_{H}=\sqrt{\frac{H(2 H-1)}{\beta\left(2-2 H, H-\frac{1}{2}\right)}}$ and $\beta($,$) denotes the Beta function.$ We put $K^{H}(t, s)=0$ if $t \leq s$ and $d(H)=\frac{1}{H+1} \sqrt{\frac{H}{2(2 H-1)}}$ is a normalizing constant.

The covariance of the Rosenblatt process $\left\{Z_{H}(t), t \in[0, T]\right\}$ satisfies, for every $s, t \geq 0$,

$$
R_{H}(s, t):=\left(Z_{H}(t) Z_{H}(s)\right)=\frac{1}{2}\left(t^{2 H}+s^{2 H}-|t-s|^{2 H}\right) .
$$

The basic observation is the fact that the covariance structure of the Rosenblatt process is similar to the one of the fractional Brownian motion and this allows the use of the same classes of deterministic integrands as in the fractional Brownian motion case whose properties are known. 
Now, we introduce Wiener integrals with respect to the Rosenblatt process. We refer to [22] for additional details on the Rosenblatt process .

By formula (2.6) we can write

$\mathrm{Z}_{H}(t)=\int_{0}^{t} \int_{0}^{t} I\left(1_{[0, t]}\right)\left(y_{1}, y_{2}\right) d B\left(y_{1}\right) d B\left(y_{2}\right)$,

where by $I$ we denote the mapping on the set of functions $f:[0, T] \longrightarrow \mathbf{R}$ to the set of functions $g:[0, T]^{2} \longrightarrow \mathbf{R}$

$$
I(f)\left(y_{1}, y_{2}\right)=d(H) \int_{y_{1} \vee y_{2}}^{T} f(u) \frac{\partial K^{H^{\prime}}}{\partial u}\left(u, y_{1}\right) \frac{\partial K^{H^{\prime}}}{\partial u}\left(u, y_{2}\right) d u .
$$

Let us denote by $\mathcal{E}$ the class of elementary functions on $\mathbf{R}$ of the form

$$
f(.)=\sum_{j=1}^{n} a_{j} 1_{\left(t_{j}, t_{j+1}\right]}(.), \quad 0 \leq t_{j}<t_{j+1} \leq T, \quad a_{j} \in \mathbf{R}, \quad i=1, \ldots, n .
$$

For $f \in \mathcal{E}$ as above, it is natural to define its Wiener integral with respect to the Rosenblatt process $Z_{H}$ by

$$
\int_{0}^{T} f(s) d Z_{H}(s):=\sum_{j=1}^{n} a_{j}\left[Z_{H}\left(t_{j+1}\right)-Z_{H}\left(t_{j}\right)\right]=\int_{0}^{T} \int_{0}^{T} I(f)\left(y_{1}, y_{2}\right) d B\left(y_{1}\right) d B\left(y_{2}\right) .
$$

Let $\mathcal{H}$ be the set of functions $f$ such that

$$
\mathcal{H}=\left\{f:[0, T] \longrightarrow \mathbf{R}: \quad\|f\|_{\mathcal{H}}:=\int_{0}^{T} \int_{0}^{T}\left(I(f)\left(y_{1}, y_{2}\right)\right)^{2} d y_{1} d y_{2}<\infty\right\} .
$$

It hold that (see Maejima and Tudor [14])

$$
\|f\|_{\mathcal{H}}=H(2 H-1) \int_{0}^{T} \int_{0}^{T} f(u) f(v)|u-v|^{2 H-2} d u d v
$$

and, the mapping

$$
f \longrightarrow \int_{0}^{T} f(u) d Z_{H}(u)
$$

provides an isometry from $\mathcal{E}$ to $L^{2}(\Omega)$. On the other hand, it has been proved in [16] that the set of elementary functions $\mathcal{E}$ is dense in $\mathcal{H}$. As a consequence the mapping (2.8) can be extended to an isometry from $\mathcal{H}$ to $L^{2}(\Omega)$. We call this extension as the Wiener integral of $f \in \mathcal{H}$ with respect 
to $Z_{H}$.

Let us consider the operator $K_{H}^{*}$ from $\mathcal{E}$ to $\mathbf{L}^{2}([0, T])$ defined by

$$
\left(K_{H}^{*} \varphi\right)\left(y_{1}, y_{2}\right)=\int_{y_{1} \vee y_{2}}^{T} \varphi(r) \frac{\partial K}{\partial r}\left(r, y_{1}, y_{2}\right) d r
$$

where $K(., .,$.$) is the kernel of Rosenblatt process in representation (2.6)$

$$
K\left(r, y_{1}, y_{2}\right)=1_{[0, t]}\left(y_{1}\right) 1_{[0, t]}\left(y_{2}\right) \int_{y_{1} \vee y_{2}}^{t} \frac{\partial K^{H^{\prime}}}{\partial u}\left(u, y_{1}\right) \frac{\partial K^{H^{\prime}}}{\partial u}\left(u, y_{2}\right) d u
$$

We refer to [22] for the proof of the fact that $K_{H}^{*}$ is an isometry between $\mathcal{H}$ and $L^{2}([0, T])$. It follows from $[22]$ that $\mathcal{H}$ contains not only functions but its elements could be also distributions. In order to obtain a space of functions contained in $\mathcal{H}$, we consider the linear space $|\mathcal{H}|$ generated by the measurable functions $\psi$ such that

$$
\|\psi\|_{|\mathcal{H}|}^{2}:=\alpha_{H} \int_{0}^{T} \int_{0}^{T}|\psi(s)\|\psi(t)\| s-t|^{2 H-2} d s d t<\infty
$$

where $\alpha_{H}=H(2 H-1)$. The space $|\mathcal{H}|$ is a Banach space with the norm $\|\psi\|_{|\mathcal{H}|}$ and we have the following inclusions (see [22]).

$$
\mathbf{L}^{2}([0, T]) \subseteq \mathbf{L}^{1 / H}([0, T]) \subseteq|\mathcal{H}| \subseteq \mathcal{H},
$$

and for any $\psi \in \mathbf{L}^{2}([0, T])$, we have

$$
\|\psi\|_{|\mathcal{H}|}^{2} \leq 2 H T^{2 H-1} \int_{0}^{T}|\psi(s)|^{2} d s
$$

Let $X$ and $Y$ be two real, separable Hilbert spaces and let $\mathcal{L}(Y, X)$ be the space of bounded linear operator from $Y$ to $X$. For the sake of convenience, we shall use the same notation to denote the norms in $X, Y$ and $\mathcal{L}(Y, X)$. Let $Q \in \mathcal{L}(Y, Y)$ be an operator defined by $Q e_{n}=\lambda_{n} e_{n}$ with finite trace $\operatorname{tr} Q=\sum_{n=1}^{\infty} \lambda_{n}<\infty$. where $\lambda_{n} \geq 0(n=1,2 \ldots)$ are non-negative real numbers and $\left\{e_{n}\right\}(n=1,2 \ldots)$ is a complete orthonormal basis in $Y$. We define the infinite dimensional $Q$-Rosenblatt process on $Y$ as

$$
Z_{H}(t)=Z_{Q}(t)=\sum_{n=1}^{\infty} \sqrt{\lambda_{n}} e_{n} z_{n}(t)
$$


where $\left(z_{n}\right)_{n \geq 0}$ is a family of real independent Rosenblatt process.

Note that the series (2.9) is convergent in $L^{2}(\Omega)$ for every $t \in[0, T]$, since

$$
\left\|Z_{Q}(t)\right\|^{2}=\sum_{n=1}^{\infty} \lambda_{n}\left(z_{n}(t)\right)^{2}=t^{2 H} \sum_{n=1}^{\infty} \lambda_{n}<\infty .
$$

Note also that $Z_{Q}$ has covariance function in the sense that

$$
E\left\langle Z_{Q}(t), x\right\rangle\left\langle Z_{Q}(s), y\right\rangle=R(s, t)\langle Q(x), y\rangle \text { for all } x, y \in Y \text { and } t, s \in[0, T] \text {. }
$$

In order to define Wiener integrals with respect to the $Q$-Rosenblatt process, we introduce the space $\mathcal{L}_{2}^{0}:=\mathcal{L}_{2}^{0}(Y, X)$ of all $Q$-Hilbert-Schmidt operators $\psi: Y \rightarrow X$. We recall that $\psi \in \mathcal{L}(Y, X)$ is called a $Q$-HilbertSchmidt operator, if

$$
\|\psi\|_{\mathcal{L}_{2}^{0}}^{2}:=\sum_{n=1}^{\infty}\left\|\sqrt{\lambda_{n}} \psi e_{n}\right\|^{2}<\infty
$$

and that the space $\mathcal{L}_{2}^{0}$ equipped with the inner product $\langle\varphi, \psi\rangle_{\mathcal{L}_{2}^{0}}=\sum_{n=1}^{\infty}\left\langle\varphi e_{n}, \psi e_{n}\right\rangle$ is a separable Hilbert space.

Now, let $\phi(s) ; s \in[0, T]$ be a function with values in $\mathcal{L}_{2}^{0}(Y, X)$, such that $\sum_{n=1}^{\infty}\left\|K^{*} \phi Q^{\frac{1}{2}} e_{n}\right\|_{\mathcal{L}_{2}^{0}}^{2}<\infty$. The Wiener integral of $\phi$ with respect to $Z_{Q}$ is defined by

$$
\begin{aligned}
& \int_{0}^{t} \phi(s) d Z_{Q}(s)=\sum_{n=1}^{\infty} \int_{0}^{t} \sqrt{\lambda_{n}} \phi(s) e_{n} d z_{n}(s) \\
= & \sum_{n=1}^{\infty} \int_{0}^{t} \int_{0}^{t} \sqrt{\lambda_{n}} K_{H}^{*}\left(\phi e_{n}\right)\left(y_{1}, y_{2}\right) d B\left(y_{1}\right) d B\left(y_{2}\right) .
\end{aligned}
$$

Now, we end this subsection by stating the following result which is fundamental to prove our result.

If $\psi:[0, T] \rightarrow \mathcal{L}_{2}^{0}(Y, X)$ satisfies $\int_{0}^{T}\|\psi(s)\|_{\mathcal{L}_{2}^{0}}^{2} d s<\infty$ then the above sum in (2.10) is well defined as a $X$-valued random variable and we have

$$
\mathbf{E}\left\|\int_{0}^{t} \psi(s) d Z_{H}(s)\right\|^{2} \leq 2 H t^{2 H-1} \int_{0}^{t}\|\psi(s)\|_{\mathcal{L}_{2}^{0}}^{2} d s .
$$


Proof. By Lemma 2.2, we have

$$
\begin{aligned}
\mathbf{E}\left\|\int_{0}^{t} \psi(s) d Z_{H}(s)\right\|^{2} & =\sum_{n=1}^{\infty} \mathbf{E}\left\|\int_{0}^{t} \int_{0}^{t} \sqrt{\lambda_{n}} K_{H}^{*}\left(\psi e_{n}\right)\left(y_{1}, y_{2}\right) d B_{n}\left(y_{1}\right) d B_{n}\left(y_{2}\right)\right\|^{2} \\
& \leq \sum_{n=1}^{\infty} 2 H t^{2 H-1} \int_{0}^{t} \lambda_{n}\left\|\psi(s) e_{n}\right\|^{2} d s \\
& =2 H t^{2 H-1} \int_{0}^{t}\|\psi(s)\|_{\mathcal{L}_{2}^{0}}^{2} d s .
\end{aligned}
$$

\subsection{Definition and assumption}

Henceforth we will assume that the family $\{A(t), t \in[0, T]\}$ of linear operators generates an evolution system of operators $\{U(t, s), 0 \leq s \leq t \leq T\}$.

An $X$-valued stochastic process $\{x(t), t \in[-\tau, T]\}$, is called a mild solution of equation (1.1) if

i) $x(.) \in \mathcal{C}\left([-\tau, T], \mathbf{L}^{2}(\Omega, X)\right)$,

ii) $x(t)=\varphi(t),-\tau \leq t \leq 0$.

iii) For arbitrary $t \in[0, T], x(t)$ satisfies the following integral equation:

$$
\begin{aligned}
x(t) & =U(t, 0)(\varphi(0)+g(0, \varphi(-r(0))))-g(t, x(t-r(t))) \\
& -\int_{0}^{t} U(t, s) A(s) g(s, x(s-r(s))) d s+\int_{0}^{t} U(t, s) f(s, x(s-\rho(s))) d s \\
& +\int_{0}^{t} U(t, s) \sigma(s) d Z_{Q}(s) \quad \mathbf{P}-a . s
\end{aligned}
$$

We introduce the following assumptions:

$(\mathcal{H} .1) \quad$ i) The evolution family is exponentially stable, that is, there exist two constants $\beta>0$ and $M \geq 1$ such that

$$
\|U(t, s)\| \leq M e^{-\beta(t-s)}, \quad \text { for all } \quad t \geq s,
$$

ii) There exist a constant $M_{*}>0$ such that

$$
\left\|A^{-1}(t)\right\| \leq M_{*} \quad \text { for all } \quad t \in[0, T] .
$$


$(\mathcal{H} .2)$ The maps $f, g:[0, T] \times X \rightarrow X$ are continuous functions and there exist two positive constants $C_{1}$ and $C_{2}$, such that for all $t \in[0, T]$ and $x, y \in X:$

i) $\|f(t, x)-f(t, y)\| \vee\|g(t, x)-g(t, y)\| \leq C_{1}\|x-y\|$.

ii) $\|f(t, x)\|^{2} \vee\left\|A^{k}(t) g(t, x)\right\|^{2} \leq C_{2}\left(1+\|x\|^{2}\right), \quad k=0,1$.

$(\mathcal{H} .3) \quad$ i) There exists a constant $0<L_{*}<\frac{1}{M_{*}}$ such that

$$
\|A(t) g(t, x)-A(t) g(t, y)\| \leq L_{*}\|x-y\|,
$$

for all $t \in[0, T]$ and $x, y \in X$.

ii) The function $g$ is continuous in the quadratic mean sense: for all $x(.) \in \mathcal{C}\left([0, T], L^{2}(\Omega, X)\right)$, we have

$$
\lim _{t \longrightarrow s}\|g(t, x(t))-g(s, x(s))\|^{2}=0 .
$$

$(\mathcal{H} .4) \quad i)$ The map $\sigma:[0, T] \longrightarrow \mathcal{L}_{2}^{0}(Y, X)$ is bounded, that is : there exists a positive constant $L$ such that $\|\sigma(t)\|_{\mathcal{L}_{2}^{0}(Y, X)} \leq L$ uniformly in $t \in[0, T]$.

ii) Moreover, we assume that the initial data $\varphi=\{\varphi(t):-\tau \leq t \leq$ $0\}$ satisfies $\varphi \in \mathcal{C}\left([-\tau, 0], \mathbf{L}^{2}(\Omega, X)\right)$.

\section{Existence and Uniqueness of Mild Solutions}

In this section we study the existence and uniqueness of mild solutions of equation (1.1). First, it is of great importance to establish the basic properties of the stochastic convolution integral of the form

$$
X(t)=\int_{0}^{t} U(t, s) \sigma(s) d Z_{Q}(s), \quad t \in[0, T]
$$

where $\sigma(s) \in \mathcal{L}_{2}^{0}(Y, X)$ and $\{U(t, s), 0 \leq s \leq t \leq T\}$ is an evolution system of operators.

The properties of the process $X$ are crucial when regularity of the mild solution to stochastic evolution equation is studied, see [5] for asystematic account of the theory of mild solutions to infinite-dimensional stochastic 
equations. Unfortunately, the process $X$ is not a martingale, and standard tools of the martingale theory, yielding e.g. continuity of the trajectories or $\mathbf{L}^{2}$-estimates are not available. The following result on the stochastic convolution integral $X$ holds. Suppose that $\sigma:[0, T] \rightarrow \mathcal{L}_{2}^{0}(Y, X)$ satisfies $\sup _{t \in[0, T]}\|\sigma(t)\|_{\mathcal{L}_{2}^{0}}^{2}<\infty$, and suppose that $\{U(t, s), 0 \leq s \leq t \leq T\}$ is an evolution system of operators satisfying $\|U(t, s)\| \leq M e^{-\beta(t-s)}$, for some constants $\beta>0$ and $M \geq 1$ for all $t \geq s$. Then, we have

1. The stochastic integral $X: t \longrightarrow \int_{0}^{t} U(t, s) \sigma(s) d Z_{Q}(s)$ is welldefined and for any $p \geq 2$ we have

$$
\mathbf{E}\left\|\int_{0}^{t} U(t, s) \sigma(s) d Z_{Q}(s)\right\|^{p} \leq C t^{p H}\left(\sup _{t \in[0, T]}\|\sigma(t)\|_{\mathcal{L}_{2}^{0}}\right)^{p},
$$

where $C$ is a constant depending only on $H, M, p$ and $\beta$.

2. The stochastic integral $X: \quad t \longrightarrow \int_{0}^{t} U(t, s) \sigma(s) d Z_{Q}(s)$ is continuous.

Proof. 1. Using the Kahane-Khintchine inequality, there exists a constant $C_{p}$ such that

$$
\mathbf{E}\left\|\int_{0}^{t} U(t, s) \sigma(s) d Z_{Q}(s)\right\|^{p} \leq C_{p}\left(\mathbf{E}\left\|\int_{0}^{t} U(t, s) \sigma(s) d Z_{Q}(s)\right\|^{2}\right)^{\frac{p}{2}} .
$$

Let $\left\{e_{n}\right\}_{n \in}$ be the complete orthonormal basis of $Y$ and $\left\{z_{n}\right\}_{n \in}$ is a sequence of independent, real-valued Rosenblatt process each with the same parameter $H \in\left(\frac{1}{2}, 1\right)$. Thus, using isometry property one can write

$$
\begin{aligned}
\mathbf{E}\left\|\int_{0}^{t} U(t, s) \sigma(s) d Z_{Q}(s)\right\|^{2} & =\sum_{n=1}^{\infty} \mathbf{E}\left\|\int_{0}^{t} U(t, s) \sigma(s) e_{n} d z_{n}(s)\right\|^{2} \\
& =H(2 H-1) \int_{0}^{t}\{\|U(t, s) \sigma(s)\| \\
& \left.\times \int_{0}^{t}\|U(t, r) \sigma(r)\||s-r|^{2 H-2} d r\right\} d s \\
& \leq H(2 H-1) M^{2} \int_{0}^{t}\left\{e^{-\beta(t-s)}\|\sigma(s)\|_{\mathcal{L}_{2}^{0}}\right. \\
& \left.\times \int_{0}^{t} e^{-\beta(t-r)}|s-r|^{2 H-2}\|\sigma(r)\|_{\mathcal{L}_{2}^{0}} d r\right\} d s .
\end{aligned}
$$


Since $\sigma$ is bounded, one can then conclude that

$$
\begin{aligned}
\mathbf{E}\left\|\int_{0}^{t} U(t, s) \sigma(s) d Z_{H}(s)\right\|^{2} & \leq H(2 H-1) M^{2}\left(\sup _{t \in[0, T]}\|\sigma(t)\|_{\mathcal{L}_{2}^{0}}\right)^{2} \int_{0}^{t}\left\{e^{-\beta(t-s)}\right. \\
& \left.\times \int_{0}^{t} e^{-\beta(t-r)}|s-r|^{2 H-2} d r\right\} d s .
\end{aligned}
$$

Make the following change of variables, $v=t-s$ for the first integral and $u=t-r$ for the second. One can write

$$
\begin{aligned}
\mathbf{E}\left\|\int_{0}^{t} U(t, s) \sigma(s) d Z_{H}(s)\right\|^{2} & \leq H(2 H-1) M^{2}\left(\sup _{t \in[0, T]}\|\sigma(t)\|_{\mathcal{L}_{2}^{0}}\right)^{2} \int_{0}^{t}\left\{e^{-\beta v}\right. \\
& \left.\times \int_{0}^{t} e^{-\beta u}|u-v|^{2 H-2} d u\right\} d v \\
& \leq H(2 H-1) M^{2}\left(\sup _{t \in[0, T]}\|\sigma(t)\|_{\mathcal{L}_{2}^{0}}\right)^{2} \int_{0}^{t} \int_{0}^{t}|u-v|^{2 H-2} d u d v .
\end{aligned}
$$

By using the equality,

$$
R_{H}(t, s)=H(2 H-2) \int_{0}^{t} \int_{0}^{s}|u-v|^{2 H-2} d u d v,
$$

we get that

$$
\mathbf{E}\left\|\int_{0}^{t} U(t, s) \sigma(s) d Z_{Q}(s)\right\|^{2} \leq C_{H} M^{2} t^{2 H}\left(\sup _{t \in[0, T]}\|\sigma(t)\|_{\mathcal{L}_{2}^{0}}\right)^{2} .
$$

Thus we obtain

$$
\mathbf{E}\left\|\int_{0}^{t} U(t, s) \sigma(s) d Z_{Q}(s)\right\|^{p} \leq C t^{p H}\left(\sup _{t \in[0, T]}\|\sigma(t)\|_{\mathcal{L}_{2}^{0}}\right)^{p}
$$

2. Let $h>0$ small enough, we have

$$
\begin{aligned}
\left\|\int_{0}^{t+h} U(t+h, s) \sigma(s) d Z_{Q}(s)-\int_{0}^{t} U(t, s) \sigma(s) d Z_{Q}(s)\right\|^{2} & \leq 2\left\|\int_{0}^{t}(U(t+h, s)-U(t, s)) \sigma(s) d Z_{Q}(s)\right\|^{2} \\
& +2\left\|\int_{t}^{t+h} U(t+h, s) \sigma(s) d Z_{H}(s)\right\|^{2} \\
& \leq 2\left[\left\|I_{1}(h)\right\|^{2}+\left\|I_{2}(h)\right\|^{2}\right] .
\end{aligned}
$$


By Lemma 2.2, we get that

$$
E\left\|I_{1}(h)\right\|^{2} \leq 2 H t^{2 H-1} \int_{0}^{t}\|[U(t+h, s)-U(t, s)] \sigma(s)\|_{\mathcal{L}_{2}^{0}}^{2} d s .
$$

Since

$$
\lim _{h \rightarrow 0}\|[U(t+h, s)-U(t, s)] \sigma(s)\|_{\mathcal{L}_{2}^{0}}^{2}=0
$$

and

$$
\|(U(t+h, s)-U(t, s)) \sigma(s)\|_{\mathcal{L}_{2}^{0}} \leq M L e^{-\beta(t-s)} e^{-\beta h+1} \in \mathbf{L}^{1}([0, T], d s),
$$

we conclude, by the dominated convergence theorem that,

$$
\lim _{h \rightarrow 0} \mathbf{E}\left\|I_{1}(h)\right\|^{2}=0 .
$$

Again by Lemma 2.2, we get that

$$
\mathbf{E}\left\|I_{2}(h)\right\|^{2} \leq \frac{2 H t^{2 H-1} L M^{2}\left(1-e^{-2 \beta h}\right)}{2 \beta} .
$$

Thus,

$$
\lim _{h \rightarrow 0} \mathbf{E}\left\|I_{2}(h)\right\|^{2}=0
$$

Thanks to Lemma 3, the stochastic integral $X(t)$ is well-defined and it belongs to the space $\mathcal{C}\left([-\tau, 0], \mathbf{L}^{2}(\Omega, X)\right)$.

We have the following theorem on the existence and uniqueness of mild solutions of equation (1.1).

Suppose that $(\mathcal{H} .1)-(\mathcal{H} .4)$ hold. Then, for all $T>0$, the equation (1.1) has a unique mild solution on $[-\tau, T]$.

Proof. Fix $T>0$ and let $B_{T}:=\mathcal{C}\left([-\tau, T], \mathbf{L}^{2}(\Omega, X)\right)$ be the Banach space of all continuous functions from $[-\tau, T]$ into $\mathbf{L}^{2}(\Omega, X)$, equipped with the supremum norm

$$
\|x\|_{B_{T}}^{2}=\sup _{-\tau \leq t \leq T} \mathbf{E}\|x(t, \omega)\|^{2} .
$$

Let us consider the set

$$
S_{T}(\varphi)=\left\{x \in B_{T}: x(s)=\varphi(s), \text { for } s \in[-\tau, 0]\right\}
$$


$S_{T}(\varphi)$ is a closed subset of $B_{T}$ provided with the norm $\|\cdot\|_{B_{T}}$.

We transform (1.1) into a fixed-point problem. Consider the operator $\psi$ on $S_{T}(\varphi)$ defined by $\psi(x)(t)=\varphi(t)$ for $t \in[-\tau, 0]$ and for $t \in[0, T]$

$$
\begin{aligned}
\psi(x)(t) & =U(t, 0)(\varphi(0)+g(0, \varphi(-r(0))))-g(t, x(t-r(t))) \\
& -\int_{0}^{t} U(t, s) A(s) g(s, x(s-r(s))) d s+\int_{0}^{t} U(t, s) f(s, x(s-\rho(s))) d s \\
& +\int_{0}^{t} U(t, s) \sigma(s) d Z_{Q}(s) \\
& =\sum_{i=1}^{5} I_{i}(t) .
\end{aligned}
$$

Clearly, the fixed points of the operator $\psi$ are mild solutions of (1.1). The fact that $\psi$ has a fixed point will be proved in several steps. We will first prove that the function $\psi$ is well defined.

Step 1: For arbitrary $x \in S_{T}(\varphi)$, we are going to show that each function $t \rightarrow I_{i}(t)$ is continuous on $[0, T]$ in the $\mathbf{L}^{2}(\Omega, X)$-sense.

For the first term $I_{1}(h)$, by Definition 2.1, we obtain

$$
\lim _{h \longrightarrow 0}(U(t+h, 0)-U(t, 0))(\varphi(0)+g(0, \varphi(-r(0))))=0 .
$$

From $(\mathcal{H} .1)$, we have

$$
\begin{gathered}
\|(U(t+h, 0)-U(t, 0))(\varphi(0)+g(0, \varphi(-r(0))))\| \\
\leq M e^{-\beta t}\left(e^{-\beta h}+1\right)\|\varphi(0)+g(0, \varphi(-r(0)))\| \in L^{2}(\Omega) .
\end{gathered}
$$

Then we conclude by the Lebesgue dominated theorem that

$$
\lim _{h \longrightarrow 0}\left\|I_{1}(t+h)-I_{1}(t)\right\|^{2}=0 .
$$

For the second term $I_{2}(h)$, assumption $(\mathcal{H} .2)$ ensures that

$$
\lim _{h \longrightarrow 0}\left\|I_{2}(t+h)-I_{2}(t)\right\|^{2}=0 .
$$

To show that the third term $I_{3}(h)$ is continuous, we suppose $h>0$ (similar calculus for $h<0$ ). We have 


$$
\begin{aligned}
\left\|I_{3}(t+h)-I_{3}(t)\right\| \leq & \left\|\int_{0}^{t}(U(t+h, s)-U(t, s)) A(s) g(s, x(s-r(s))) d s\right\| \\
& +\left\|\int_{t}^{t+h} U(t, s) g(s, x(s-r(s))) d s\right\| \\
\leq & I_{31}(h)+I_{32}(h) .
\end{aligned}
$$

By Hölder's inequality, we have

$$
\left\|I_{31}(h)\right\| \leq t \int_{0}^{t} \|(U(t+h, s)-U(t+h, s)) A(s) g\left(s, x(s-r(s)) \|^{2} d s .\right.
$$

By Definition 2.1, we obtain

$$
\lim _{h \longrightarrow 0}(U(t+h, s)-U(t, s)) A(s) g(s, x(s-r(s)))=0 .
$$

From $(\mathcal{H} .1)$ and $(\mathcal{H} .2)$, we have

$$
\begin{gathered}
\|(U(t+h, s)-U(t, s)) A(s) g(s, x(s-r(s)))\| \\
\leq C_{2} M e^{-\beta(t-s)}\left(e^{-\beta h}+1\right)\|A(s) g(s, x(s-r(s)))\| \in L^{2}(\Omega) .
\end{gathered}
$$

Then we conclude by the Lebesgue dominated theorem that

$$
\lim _{h \longrightarrow 0}\left\|I_{31}(h)\right\|^{2}=0 .
$$

So, estimating as before. By using $(\mathcal{H} .1)$ and $(\mathcal{H} .2)$, we get

$$
\left\|I_{32}(h)\right\|^{2} \leq \frac{M^{2} C_{2}\left(1-e^{-2 \beta h}\right)}{2 \beta} \int_{t}^{t+h}\left(1+\|x(s-r(s))\|^{2}\right) d s .
$$

Thus,

$$
\lim _{h \longrightarrow 0}\left\|I_{32}(h)\right\|^{2}=0 .
$$

For the fourth term $I_{4}(h)$, we suppose $h>0$ (similar calculus for $\left.h<0\right)$. We have

$$
\begin{aligned}
\left\|I_{4}(t+h)-I_{4}(t)\right\| \leq & \left\|\int_{0}^{t}(U(t+h, s)-U(t, s)) f(s, x(s-\rho(s))) d s\right\| \\
& +\left\|\int_{t}^{t+h} U(t, s) f(s, x(s-\rho(s))) d s\right\| \\
\leq & \left\|I_{41}(h)\right\|+\left\|I_{42}(h)\right\| .
\end{aligned}
$$


By Hölder's inequality, we have

$$
\left\|I_{41}(h)\right\| \leq t \int_{0}^{t}\|(U(t+h, s)-U(t, s)) f(s, x(s-\rho(s)))\|^{2} d s .
$$

Again exploiting properties of Definition 2.1, we obtain

$$
\lim _{h \longrightarrow 0}(U(t+h, s)-U(t, s)) f(s, x(s-\rho(s)))=0,
$$

and

$$
\begin{gathered}
\|(U(t+h, s)-U(t, s)) f(s, x(s-\rho(s)))\| \\
\leq M e^{-\beta(t-s)}\left(e^{-\beta h}+1\right)\|f(s, x(s-\rho(s)))\| \in L^{2}(\Omega) .
\end{gathered}
$$

Then we conclude by the Lebesgue dominated theorem that

$$
\lim _{h \longrightarrow 0}\left\|I_{41}(h)\right\|^{2}=0 .
$$

On the other hand, by $(\mathcal{H} .1),(\mathcal{H} .2)$, and the Hölder's inequality, we have

$$
\left\|I_{42}(h)\right\| \leq \frac{M^{2} C_{2}\left(1-e^{-2 \beta h}\right)}{2 \beta} \int_{t}^{t+h}\left(1+\|x(s-\rho(s))\|^{2}\right) d s .
$$

Thus

$$
\lim _{h \rightarrow 0}\left\|I_{42}(h)\right\|^{2}=0 .
$$

Now, for the term $I_{5}(h)$, we have

$$
\begin{aligned}
\left\|I_{5}(t+h)-I_{5}(t)\right\|^{2} & \leq 2\left\|\int_{0}^{t}(U(t+h, s)-U(t, s)) \sigma(s) d Z_{Q}(s)\right\|^{2} \\
& +2\left\|\int_{t}^{t+h} U(t+h, s) \sigma(s) d Z_{Q}(s)\right\|^{2}
\end{aligned}
$$

By Lemma 3 we get

$$
\lim _{h \rightarrow 0}\left\|I_{5}(t+h)-I_{5}(t)\right\|^{2}=0 .
$$

The above arguments show that $\lim _{h \rightarrow 0} \mathbf{E}\|\psi(x)(t+h)-\psi(x)(t)\|^{2}=0$. Hence, we conclude that the function $t \rightarrow \psi(x)(t)$ is continuous on $[0, T]$ in the $\mathbf{L}^{2}$-sense. 
Step 2: Now, we are going to show that $\psi$ is a contraction mapping in $S_{T_{1}}(\varphi)$ with some $T_{1} \leq T$ to be specified later. Let $x, y \in S_{T}(\varphi)$, by using the inequality

$$
(a+b+c)^{2} \leq \frac{1}{\nu} a^{2}+\frac{2}{1-\nu} b^{2}+\frac{2}{1-\nu} c^{2},
$$

where $\nu:=L_{*} M_{*}<1$, we obtain for any fixed $t \in[0, T]$

$$
\begin{aligned}
\| \psi(x)(t)- & \psi(y)(t) \|^{2} \\
\leq & \frac{1}{\nu}\|g(t, x(t-r(t)))-g(t, y(t-r(t)))\|^{2} \\
& +\frac{2}{1-\nu}\left\|\int_{0}^{t} U(t, s) A(s)(g(s, x(s-r(s)))-g(s, y(s-r(s)))) d s\right\|^{2} \\
& +\frac{2}{1-\nu}\left\|\int_{0}^{t} U(t, s)(f(s, x(s-\rho(s)))-f(s, y(s-\rho(s)))) d s\right\|^{2} \\
= & \sum_{k=1}^{3} J_{k}(t) .
\end{aligned}
$$

By using the fact that the operator $\left\|\left(A^{-1}(t)\right)\right\|$ is bounded, combined with the condition $(\mathcal{H} .3)$, we obtain that

$$
\begin{aligned}
\left\|J_{1}(t)\right\| & \leq \frac{1}{\nu}\left\|A^{-1}(t)\right\|^{2} \mid A(t) g(t, x(t-r(t)))-A(t) g(t, y(t-r(t))) \|^{2} \\
& \leq \frac{L_{*}^{2} M_{*}^{2}}{\nu} \mathbf{E}\|x(t-r(t))-y(t-r(t))\|^{2} \\
& \leq \nu \sup _{s \in[-\tau, t]} \mathbf{E}\|x(s)-y(s)\|^{2} .
\end{aligned}
$$

By hypothesis ( $\mathcal{H} .3)$ combined with Hölder's inequality, we get that

$$
\begin{aligned}
\left\|J_{2}(t)\right\| & \leq\left\|\int_{0}^{t} U(t, s)[A(t) g(t, x(t-r(t)))-A(t) g(t, y(t-r(t)))] d s\right\| \\
& \leq \frac{2}{1-\nu} \int_{0}^{t} M^{2} e^{-2 \beta(t-s)} d s \int_{0}^{t} \mathbf{E}\|x(s-r(s))-y(s-r(s))\|^{2} d s \\
& \leq \frac{2 M^{2} L_{*}^{2}}{1-\nu} \frac{1-e^{-2 \beta t}}{2 \beta} t \sup _{s \in[-\tau, t]} \mathbf{E}\|x(s)-y(s)\|^{2} .
\end{aligned}
$$


Moreover, by hypothesis $(\mathcal{H} .2)$ combined with Hölder's inequality, we can conclude that

$$
\begin{aligned}
E\left\|J_{3}(t)\right\| & \leq E\left\|\int_{0}^{t} U(t, s)[f(s, x(s-\rho(s)))-f(s, y(s-\rho(s)))] d s\right\|^{2} \\
& \leq \frac{2 C_{1}^{2}}{1-\nu} \int_{0}^{t} M^{2} e^{-2 \beta(t-s)} d s \int_{0}^{t} \mathbf{E}\|x(s-r(s))-y(s-r(s))\|^{2} d s \\
& \leq \frac{2 M^{2} C_{1}^{2}}{1-\nu} \frac{1-e^{-2 \beta t}}{2 \beta} t \sup _{s \in[-\tau, t]} \mathbf{E}\|x(s)-y(s)\|^{2} .
\end{aligned}
$$

Hence

$$
\sup _{s \in[-\tau, t]} \mathbf{E}\|\psi(x)(s)-\psi(y)(s)\|^{2} \leq \gamma(t) \sup _{s \in[-\tau, t]} \mathbf{E}\|x(s)-y(s)\|^{2},
$$

where

$$
\gamma(t)=\nu+\left[L_{*}^{2}+C_{1}^{2}\right] \frac{2 M^{2}}{1-\nu} \frac{1-e^{-2 \beta t}}{2 \beta} t
$$

By condition $(\mathcal{H} .3)$, we have $\gamma(0)=\nu=L_{*} M_{*}<1$. Then there exists $0<T_{1} \leq T$ such that $0<\gamma\left(T_{1}\right)<1$ and $\psi$ is a contraction mapping on $S_{T_{1}}(\varphi)$ and therefore has a unique fixed point, which is a mild solution of equation (1.1) on $\left[-\tau, T_{1}\right]$. This procedure can be repeated in order to extend the solution to the entire interval $[-\tau, T]$ in finitely many steps. This completes the proof.

\section{An Example}

In recent years, the interest in neutral systems has been growing rapidly due to their successful applications in practical fields such as physics, chemical technology, bioengineering, and electrical networks. We consider the following stochastic partial neutral functional differential equation with finite delays $\tau_{1}$ and $\tau_{2}\left(0 \leq \tau_{i} \leq \tau<\infty, i=1,2\right)$, driven by a Rosenblatt process 


$$
\left\{\begin{aligned}
& d\left[u(t, \zeta)+G\left(t, u\left(t-\tau_{1}, \zeta\right)\right)\right]=\left[\frac{\partial^{2}}{\partial^{2} \zeta} u(t, \zeta)+b(t, \zeta) u(t, \zeta)+F\left(t, u\left(t-\tau_{2}, \zeta\right)\right)\right] d t \\
&+\sigma(t) d Z_{H}(t), 0 \leq t \leq T, 0 \leq \zeta \leq \pi, \\
& u(t, 0)=u(t, \pi)=0, \quad 0 \leq t \leq T \\
& u(t, \zeta)=\varphi(t, \zeta), \quad t \in[-\tau, 0], 0 \leq \zeta \leq \pi,
\end{aligned}\right.
$$

where $Z_{H}$ is a Rosenblatt process, $b(t, \zeta)$ is a continuous function and is uniformly Hölder continuous in $t, F, G: \mathbf{R}^{+} \times \mathbf{R} \longrightarrow \mathbf{R}$ are continuous functions.

To study this system, we consider the space $X=L^{2}([0, \pi]), Y=\mathbf{R}$ and the operator $A: D(A) \subset X \longrightarrow X$ given by $A y=y^{\prime \prime}$ with

$$
D(A)=\left\{y \in X: y^{\prime \prime} \in X, \quad y(0)=y(\pi)=0\right\} .
$$

It is well known that $A$ is the infinitesimal generator of an analytic semigroup $\{T(t)\}_{t \geq 0}$ on $X$. Furthermore, $A$ has discrete spectrum with eigenvalues $-n^{2}, n \in$ and the corresponding normalized eigenfunctions given by

$$
e_{n}:=\sqrt{\frac{2}{\pi}} \sin n x, n=1,2, \ldots
$$

In addition $\left(e_{n}\right)_{n \in}$ is a complete orthonormal basis in $X$ and

$$
T(t) x=\sum_{n=1}^{\infty} e^{-n^{2} t}<x, e_{n}>e_{n}
$$

for $x \in X$ and $t \geq 0$.

Now, we define an operator $A(t): D(A) \subset X \longrightarrow X$ by

$$
A(t) x(\zeta)=A x(\zeta)+b(t, \zeta) x(\zeta) .
$$

By assuming that $b(.,$.$) is continuous and that b(t, \zeta) \leq-\gamma(\gamma>0)$ for every $t \in \mathbf{R}, \zeta \in[0, \pi]$, it follows that the system

$$
\left\{\begin{array}{l}
u^{\prime}(t)=A(t) u(t), \quad t \geq s \\
u(s)=x \in X
\end{array}\right.
$$


has an associated evolution family given by

$$
U(t, s) x(\zeta)=\left[T(t-s) \exp ^{\int_{s}^{t} b(\tau, \zeta) d \tau} x\right](\zeta) .
$$

From this expression, it follows that $U(t, s)$ is a compact linear operator and that for every $s, t \in[0, T]$ with $t>s$

$$
\|U(t, s)\| \leq e^{-(\gamma+1)(t-s)}
$$

In addition, $A(t)$ satisfies the assumption $\mathcal{H}_{1}$ (see [2]).

To rewrite the initial-boundary value problem (4.1) in the abstract form we assume the following:

i) The substitution operator $f:[0, T] \times X \longrightarrow X$ defined by $f(t, u)()=$. $F(t, u()$.$) is continuous and we impose suitable conditions on \mathrm{F}$ to verify assumption $\mathcal{H}_{2}$.

ii) The substitution operator $g:[0, T] \times X \longrightarrow X$ defined by $g(t, u)()=$. $G(t, u()$.$) is continuous and we impose suitable conditions on \mathrm{G}$ to verify assumptions $\mathcal{H}_{2}$ and $\mathcal{H}_{3}$.

iii) The function $\sigma:[0, T] \longrightarrow \mathcal{L}_{2}^{0}\left(L^{2}([0, \pi]), \mathbf{R}\right)$ is bounded, that is, there exists a positive constant $L$ such that $\|\sigma(t)\|_{\mathcal{L}_{2}^{0}} \leq L<\infty$, uniformly in $t \in[0, T]$, where $L:=\sup _{t \in[0, T]} e^{-t}$.

If we put

$$
\left\{\begin{array}{l}
u(t)(\zeta)=u(t, \zeta), t \in[0, T], \zeta \in[0, \pi] \\
u(t, \zeta)=\varphi(t, \zeta), t \in[-\tau, 0], \zeta \in[0, \pi]
\end{array}\right.
$$

then, the problem (4.1) can be written in the abstract form

$\left\{\begin{array}{l}d[x(t)+g(t, x(t-r(t)))]=[A(t) x(t)+f(t, x(t-\rho(t)))] d t+\sigma(t) d Z_{H}(t), 0 \leq t \leq T, \\ x(t)=\varphi(t),-\tau \leq t \leq 0 .\end{array}\right.$

Furthermore, if we assume that the initial data $\varphi=\{\varphi(t):-\tau \leq t \leq 0\}$ satisfies $\varphi \in \mathcal{C}\left([-\tau, 0], \mathbf{L}^{2}(\Omega, X)\right)$, thus all the assumptions of Theorem 3 are fulfilled. Therefore, we conclude that the system (4.1) has a unique mild solution on $[-\tau, T]$. 


\section{Acknowledgements}

The author would like to thank the referee and the editor for their careful comments and valuable suggestions on this work.

\section{References}

[1] P. Acquistapace and B. Terreni. "A unified approach to abstract linear parabolic equations", Rendiconti del seminario matematico della Università di Padova, vol. 78, pp. 47-107, 1987. [On line]. Available: https://bit.ly/2n1nvOT

[2] D. Aoued and S. Baghli-Bendimerad, "Mild solutions for perturbed evolution equations with infinite state-dependent delay", Electronic journal of qualitative theory of differential equations, no. 59, pp. 1-24, Oct. 2013, doi:10.14232/ejqtde.2013.1.59.

[3] B. Boufoussi and S. Hajji, "Neutral stochastic functional differential equation driven by a fractional Brownian motion in a Hilbert space", Statistics \& probability letters, vol. 82 no. 8, pp. 15491558, Aug. 2012, doi: 10.1016/j.spl.2012.04.013.

[4] B. Boufoussi, S. Hajji, and E. Lakhel, "Functional differential equations in Hilbert spaces driven by a fractional Brownian motion", Afrika Matematika, vol. 23, no. 2, pp. 173-194, Jun. 2011, doi: 10.1007/s13370-011-0028-8.

[5] G. Da Prato and J. Zabczyk, Stochastic equations in infinite dimensions. Cambridge: Cambridge University Press, 1992, doi: $10.1017 /$ CBO9780511666223.

[6] E. Lakhel and S. Hajji. "Existence and uniqueness of mild solutions to neutral SFDEs driven by a fractional Brownian motion with nonLipschitz coefficients", Journal of numerical mathematics and stochastics, vol.7, no. 1, pp. 14-29, 2015. [On line]. Available: https://bit.ly/2nSMKDe

[7] A. Boudaoui and E. Lakhel, "Controllability of Stochastic Impulsive Neutral Functional Differential Equations Driven by Fractional Brownian Motion with Infinite Delay", Differential Equations and Dynamical Systems, vol. 26, no. 1-3, pp. 247-263, Nov. 2017, doi: 10.1007/s12591-017-0401-7.

[8] E. Lakhel and A. Tlidi, "Controllability of time-dependent neutral stochastic functional differential equations driven by a fractional Brownian motion", Journal of nonlinear sciences and applications, vol. 11, no. 06, pp. 850-863, May 2018., doi: 10.22436/jnsa.011.06.11. 
[9] B. Boufoussi, S. Hajji, and E. Lakhel, "Exponential stability of impulsive neutral stochastic functional differential equation driven by fractional Brownian motion and Poisson point processes", Afrika Matematika, vol. 29, no. 1-2, pp. 233-247, Nov. 2017., doi: 10.1007/s13370-017-0538-0.

[10] E. Lakhel, "Controllability of neutral functional differential equations driven by fractional Brownian motion with infinite delay", Nonlinear dynamics and systems theory, vol. 17, no. 3, pp. 291-302, 2017.

[11] E. Lakhel and M. Mckibben, "Existence of solutions for fractional neutral functional differential equations driven by fBm with infinite delay", Stochastics, vol. 90, no. 3, pp. 313-329, Jun. 2017, doi: 10.1080/17442508.2017.1346657.

[12] N. Leonenko and V. Anh, "Rate of convergence to the Rosenblatt distribution for additive functionals of stochastic processes with long-range dependence", Journal of applied mathematics and stochastic analysis, vol. 14, no. 1, pp. 27-46, Jan. 2001, doi: $10.1155 / \mathrm{S} 1048953301000041$.

[13] M. Maejima and C. Tudor, "On the distribution of the Rosenblatt process”, Statistics \& probability letters, vol. 83, no. 6, pp. 14901495, Jun. 2013, doi: 10.1016/j.spl.2013.02.019.

[14] M. Maejima and C. Tudor, "Wiener integrals with respect to the Hermite process and a non-central limit theorem", Stochastic analysis and applications, vol. 25, no. 5, pp. 1043-1056, Ago. 2007, doi: 10.1080/07362990701540519.

[15] A. Pazy, Semigroups of linear operators and applications to partial differential equations, vol. 44. New York, NY: Springer, 1983, doi: 10.1007/978-1-4612-5561-1.

[16] V. Pipiras and M. Taqqu, "Integration questions related to fractional Brownian motion", Probability theory and related fields, vol. 118, no. 2, pp. 251-291, Oct. 2000, doi: $10.1007 / \mathrm{s} 440-000-8016-7$.

[17] Y. Ren and D. Sun, "Second-order neutral impulsive stochastic evolution equations with delay", Journal of mathematical physics, vol. 50, no. 10, p. 102709 , Oct. 2009, doi: $10.1063 / 1.3251332$.

[18] R. Sakthivel, P. Revathi, Y. Ren, and G. Shen, "Retarded stochastic differential equations with infinite delay driven by Rosenblatt process", Stochastic analysis and applications, vol. 36, no. 2, pp. 304323, Dec. 2017., doi: 10.1080/07362994.2017.1399801.

[19] R. Rosenblatt, "Independence and dependence", in Proceedings of the Fourth Berkeley symposium on mathematical statistics and probability, 1961, vol. 2, pp. 431-443. [On line]. Available: https://bit.ly/2n6DkUw

[20] M. Taqqu, "Weak convergence to fractional brownian motion and to the Rosenblatt process", Zeitschrift für wahrscheinlichkeitstheorie und verwandte gebiete, vol. 31, no. 4, pp. 287-302, 1975, doi: 10.1007/BF00532868. 
Neutral stochastic functional differential evolution equations... 689

[21] M. Taqqu, "Convergence of integrated processes of arbitrary Hermite rank", Zeitschrift für wahrscheinlichkeitstheorie und verwandte gebiete, vol. 50, no. 1, pp. 53-83, 1979, doi: $10.1007 /$ BF00535674.

[22] C. Tudor, "Analysis of the Rosenblatt process", ESAIM: Probability and statistics, vol. 12, pp. 230-257, Jan. 2008., doi: 10.1051/ps:2007037. 